\title{
Cultivation of common bacterial species and strains from human skin, oral, and gut microbiota
}

\author{
Elizabeth Fleming ${ }^{1 \dagger}$, Victor Pabst ${ }^{1 \dagger}$, Zoe Scholar ${ }^{1}$, Ruoyun Xiong ${ }^{1}$, Anita Y. Voigt ${ }^{1}$, Wei Zhou', Amelia Hoyt ${ }^{1}$, \\ Rachel Hardy ${ }^{1}$, Anna Peterson ${ }^{2}$, Ryan Beach², Yvette Ondouah-Nzutchi', Jinhong Dong ${ }^{1}$, Lucinda Bateman ${ }^{3}$, \\ Suzanne D. Vernon ${ }^{3}$ and Julia $\mathrm{Oh}^{1^{*}}$ (D)
}

\begin{abstract}
Background: Genomics-driven discoveries of microbial species have provided extraordinary insights into the biodiversity of human microbiota. In addition, a significant portion of genetic variation between microbiota exists at the subspecies, or strain, level. High-resolution genomics to investigate species- and strain-level diversity and mechanistic studies, however, rely on the availability of individual microbes from a complex microbial consortia. High-throughput approaches are needed to acquire and identify the significant species- and strain-level diversity present in the oral, skin, and gut microbiome. Here, we describe and validate a streamlined workflow for cultivating dominant bacterial species and strains from the skin, oral, and gut microbiota, informed by metagenomic sequencing, mass spectrometry, and strain profiling.

Results: Of total genera discovered by either metagenomic sequencing or culturomics, our cultivation pipeline recovered between $18.1-44.4 \%$ of total genera identified. These represented a high proportion of the community composition reconstructed with metagenomic sequencing, ranging from 66.2-95.8\% of the relative abundance of the overall community. Fourier-Transform Infrared spectroscopy (FT-IR) was effective in differentiating genetically distinct strains compared with whole-genome sequencing, but was less effective as a proxy for genetic distance.

Conclusions: Use of a streamlined set of conditions selected for cultivation of skin, oral, and gut microbiota facilitates recovery of dominant microbes and their strain variants from a relatively large sample set. FT-IR spectroscopy allows rapid differentiation of strain variants, but these differences are limited in recapitulating genetic distance. Our data highlights the strength of our cultivation and characterization pipeline, which is in throughput, comparisons with high-resolution genomic data, and rapid identification of strain variation.
\end{abstract}

\section{Background}

Genomics-driven innovations, such as high-throughput $16 \mathrm{~S}$ ribosomal RNA (rRNA) gene and whole-genome shotgun metagenomic sequencing have been powerful drivers of discovery in a wide range of microbial

\footnotetext{
* Correspondence: julia.oh@jax.org

${ }^{\dagger}$ Elizabeth Fleming and Victor Pabst contributed equally to this work.

'The Jackson Laboratory, 10 Discovery Drive, Farmington, CT 860-837-2014, USA

Full list of author information is available at the end of the article
}

ecosystems, including those that impact human health. The blueprints created by large-scale studies have elucidated an extraordinary microbial biodiversity across individuals, geographies, ethnicities, disease states, and lifestyles [1]. Such blueprints have been critical for baseline characterizations of different ecosystems and generating hypotheses by correlative analyses with phenotypes of interest [2,3]. The logical next step for understanding host microbiome interactions are mechanistic investigations that are potentiated by findings from metagenomic

(C) The Author(s). 2021 Open Access This article is licensed under a Creative Commons Attribution 4.0 International License, which permits use, sharing, adaptation, distribution and reproduction in any medium or format, as long as you give appropriate credit to the original author(s) and the source, provide a link to the Creative Commons licence, and indicate if changes were made. The images or other third party material in this article are included in the article's Creative Commons licence, unless indicated otherwise in a credit line to the material. If material is not included in the article's Creative Commons licence and your intended use is not permitted by statutory regulation or exceeds the permitted use, you will need to obtain permission directly from the copyright holder. To view a copy of this licence, visit http://creativecommons.org/licenses/by/4.0/ The Creative Commons Public Domain Dedication waiver (http://creativecommons.org/publicdomain/zero/1.0/) applies to the data made available in this article, unless otherwise stated in a credit line to the data. 
surveys, but made possible by possessing the substrates of interest - namely, the microbes themselves.

Obtaining microbial isolates from a sample of interest has multiple values. First, sequencing isolates provides the highest quality reference genome sequences; genome reconstructions from metagenomic data can result in incomplete and fragmented genomes [4-6], as closely related genomes, low abundance genomes, and highly complex communities pose significant computational challenges that hinder accurate reconstruction of function and biodiversity [7]. Second, an isolate in hand allows experimentation and manipulation to understand its genetics, molecular and physiological mechanisms, and inter- and intra-species interactions. Third, isolates allow precise investigation of strain diversity, which is critical as individual strains of a microbial species can exhibit widely diverse phenotypes [7]. For example, most Escherichia (E.) coli strains are commensals in the human gastrointestinal tract, while some strains can cause severe disease [8]. In addition, isolates are necessary for inferring evolutionary dynamics, transmission, and lineage tracking during infectious disease outbreaks [9]. Finally, a tremendous genetic and phenotypic diversity is encoded at the strain level, rather than higher taxonomic levels, and individual disease susceptibility or severity phenotypes can be attributed to not only a common species, but unique strains $[8,10]$. Different algorithms have been developed to infer strain diversity from metagenomic datasets, e.g. [11-13], but these can vastly underestimate strain diversity. Thus, cultivation of microbiota accounting for strain diversity would facilitate investigation of patient-specific genotypes and phenotypes.

Finally, systematic methods for cultivation and recovery of microbial isolates from a sample is complicated by the notable site specificity of the human microbiome [14-16]. For example, the gut harbors the highest biodiversity, with characteristic bacteria from Bacteroides and varied lactobacilli, enterobacilli and enterococci, Bifidobacteria, Clostridia, and methanogens [14]. The oral cavity is typically populated with streptococci, Haemophilus, Prevotella, Veillonella genera, and the skin staphylococci with Corynebacterium and Cutibacterium [14]. Even within each of these body sites, significant local variation exists, such as the stomach vs. the small intestine vs. the cecum [17], oral pockets vs. the dorsum of the tongue $[18,19]$, or the moist, oily, dry, or foot sites of the skin [20].

Numerous approaches have been defined to systematically cultivate microbes from different ecosystems, with a focus particularly on the gut $[21,22]$. Extraordinary efforts have been made to increase the recovery of gut microbial biodiversity from the anaerobic environment of the gut, using up to 212 different culture conditions [21, 23], which might include a wide variety of different nutritive conditions or additives, different gas fractions, temperatures, $\mathrm{pH}$, or inhibition via antimicrobials. Microfluidics devices [24] for optimized isolation of cells, or metagenomic prediction of membrane epitopes for synthetic design of antibodies have also been used to capture microorganisms of interest [25]. Multiple, sequenced large-scale gut microbial culture collections have been recently established [21, 26-28], and these efforts have correspondingly increased the accurate annotation of metagenomic datasets. In contrast, while skin cultivation methods were prolific in the 1950s [29], there was no potential to inform recovery using metagenomic characterizations, and fewer consolidated and systematic efforts exist for human oral or skin microbial cultivation, with recent efforts primarily targeted efforts to recover microbes of interest, like Cutibacterium acnes [30] or Gram negatives [31].

Here, our ultimate goals were to define a set of userfriendly cultivation conditions that would allow us to 1 ) culture dominant microbiota from many different individuals and body sites, 2) estimate recovery based on metagenomic data, and importantly, 3) identify rapid, low-cost approaches to delineate strain diversity, which would facilitate isolate choice for more laborious and costly whole genome sequencing. We cultivated isolates from the human gut, oral cavity, and two physiologically diverse skin sites on a streamlined set of different nutritive conditions. We performed shotgun sequencing of the same sample to evaluate the proportion of microbes recovered by cultivation. Finally, we compared the ability of Fourier-Transform Infrared Spectroscopy (FT-IR) to rapidly classify and differentiate strains of common species with whole genome sequencing. Taken together, this work builds on and consolidates approaches for generating culture collections from a variety of different environments, enabling a range of follow-up genomic and phenotypic characterizations.

\section{Results \\ Sample description}

Our goal was to identify a core set of cultivation conditions for each body site that would 1) allow recovery of dominant microbes from a large number of samples, which 2) would recover strain variants of these species. In addition, we sought to evaluate how well these conditions promoted culture of the microbial diversity of the sample, as we anticipated that approaches favoring throughput would limit a comprehensive recovery of microbes from each body site. For our sample choice, we obtained human samples from the following sites: for the skin, we chose the forehead and toe web space as representatives of an oily and a moist skin site, respectively, and their microbiota differ markedly in our previous surveys $[7,15,20]$. We chose the inner cheek and 
tongue dorsum to represent the oral cavity, and stool for gut. Because different individuals can harbor markedly different microbial species and strains [14], we obtained a total of 25 samples ( 5 samples per body site) from 12 individuals. For each sample, we then performed metagenomic shotgun sequencing $\left(1.6 \pm 1.0 \times 10^{6}, 2.0 \pm 1.1 \times\right.$ $10^{6}, \quad 10.5 \pm 1.0 \times 10^{6}$ quality-controlled, human DNA dehosted reads for oral, skin, and stool, respectively, Table S1) and culturomics as described below.

\section{Cultivation conditions and species identification}

Guided by our previous metagenomic data and a literature search $[21,22,26,27,29-46]$, we compiled the aerobic and anaerobic cultivation conditions reported in Table 1 and Fig. 1. To examine the proportion of microbes recovered by these conditions, we first characterized the fungal, bacterial, and viral composition of our samples using shotgun metagenomics as it is cultureindependent and yields the most unbiased compositional reconstruction (Fig. 2, Fig. S1, Table S2). Consistent with previous reports [14, 15], Cutibacterium acnes and Corynebacterium sp. were most abundant in the oily sites of the forehead, and staphylococci and Corynebacterium sp. in the foot. In the cheek, streptococci, Rothia mucilanginosa, and Haemophilus parainfluenzae were most abundant, and in the tongue dorsum, Neisseria flavescens, Prevotella sp., Veillonella sp., and streptococci. Finally, in the stool samples, Bacteroidales and Clostridiales were most abundant.

In culturing, we recommend a rule of thumb to dilute samples $1: 10$ and 1:100 for skin sites, $1: 100$ and 1:1000 for oral sites, 1:1000 and 1:10000 for gut samples prior to plating, reflecting the low, medium, and high microbial bioburden of these sites, respectively. Dilution plates usually consisted of 1-4 dominant microbes with singletons interspersed at low density. Because of this we aimed to select $\sim 12$ microbes per plate, selecting up to 3 of each visibly unique morphology for subculturing to further purify the selected isolate, followed by MALDITOF mass spectrometry analysis for identification. MALDI-TOF accuracy at the species and genus level varies widely by taxonomy and even instrument (e.g., $84 \%$ for species, $\sim 92 \%$ for genus in a recent estimate of anaerobic bacteria [47], but up to $98 \%$ accuracy and $94 \%$ accuracy can be observed in Enterobacteriaceae and staphyloccci [48]), with disease-associated species having the deepest reference databases and thus the highest corresponding accuracy. Thus, we emphasize here the comparisons at the genus level, but also report specieslevel results, recognizing that discrimination at the species level is critical for many human-associated pathobionts, such as staphylococci, which encompass diseasecausing $S$. aureus vs. many commensal species. Overall, we obtained 15 unique genera ( 34 species) in the skin,
Table 1 Cultivation conditions

\begin{tabular}{ll}
\hline Skin cultivation conditions & \\
\hline Media & Conditions \\
\hline LB agar & Aerobic, \\
& Anaerobic, $37^{\circ} \mathrm{C}$ \\
R2A agar & Aerobic, \\
& Anaerobic, $37^{\circ} \mathrm{C}$ \\
TSA with 5\% sheep blood & Aerobic, \\
Brucella agar & Anaerobic, $37^{\circ} \mathrm{C}$ \\
BCYE agar & Aerobic, \\
& Anaerobic, $37^{\circ} \mathrm{C}$ \\
MacConkey agar & Aerobic, \\
Oral cultivation conditions & Anaerobic, $37^{\circ} \mathrm{C}$ \\
Media & Aerobic, \\
LB agar & Anaerobic, $37^{\circ} \mathrm{C}$ \\
R2A agar & \\
TSA with 5\% sheep blood & Conditions \\
Chocolate agar & Aerobic, \\
& Anaerobic, $37^{\circ} \mathrm{C}$ \\
& Aerobic, \\
& Anaerobic, $37^{\circ} \mathrm{C}$ \\
& Aerobic, \\
Anaerobic, $37^{\circ} \mathrm{C}$ \\
Stive Strep agar & Aerobic, \\
& Anaerobic, $37^{\circ} \mathrm{C}$ \\
Aerobic, \\
Anaerobic, $37^{\circ} \mathrm{C}$
\end{tabular}

Stool cultivation conditions

Culture device prior to plating onto TSA with $5 \%$ sheep blood

Direct plating onto GMM agar

$\operatorname{TSB}(3,7,14 d)+$ sheep blood (9\% final vol)

Aerobic blood bottle $(3,7,14 d)+$ rumen fluid ( $9 \%$ final vol)

Aerobic blood bottle $(3,7,14 d)+$ sheep blood ( $9 \%$ final vol)

Aerobic blood bottle (3, 7, 14d); sample filtered at $5 \mu \mathrm{m}$

$\mathrm{BHI}(3,7,14 \mathrm{~d})+$ vancomycin + colistin $(10 \mu \mathrm{g} / \mathrm{mL}$ each)

$\operatorname{TSB}(3,7,14 d)$

Anaerobic Blood bottle $(3,7,14 d)+$ sheep blood ( $9 \%$ final vol)

Anaerobic Blood bottle $(3,7,14 d)+$ rumen fluid (9\% final vol)

Anaerobic Blood bottle $(3,7,14 d)$; sample filtered at $5 \mu \mathrm{m}$

Anaerobic Blood bottle $(3,7,14 d)$; thermic shock $\left(85^{\circ} \mathrm{C}, 20 \mathrm{~min}\right)$

$\mathrm{BHI}(3,7,14 \mathrm{~d})+$ vancomycin + colistin

(10 $\mu \mathrm{g} / \mathrm{mL}$ each)

$\operatorname{TSB}(3,7,14 \mathrm{~d})+$ sheep blood (9\% final vol)
Conditions

Anaerobic, $37^{\circ} \mathrm{C}$

Aerobic, $28^{\circ} \mathrm{C}$

Aerobic, $37^{\circ} \mathrm{C}$

Aerobic, $37^{\circ} \mathrm{C}$

Aerobic, $37^{\circ} \mathrm{C}$

Aerobic, $37^{\circ} \mathrm{C}$

Aerobic, $37^{\circ} \mathrm{C}$

Anaerobic, $37^{\circ} \mathrm{C}$

Anaerobic, $37^{\circ} \mathrm{C}$

Anaerobic, $37^{\circ} \mathrm{C}$

Anaerobic, $37^{\circ} \mathrm{C}$

Anaerobic, $37^{\circ} \mathrm{C}$

Anaerobic, $37^{\circ} \mathrm{C}$ 


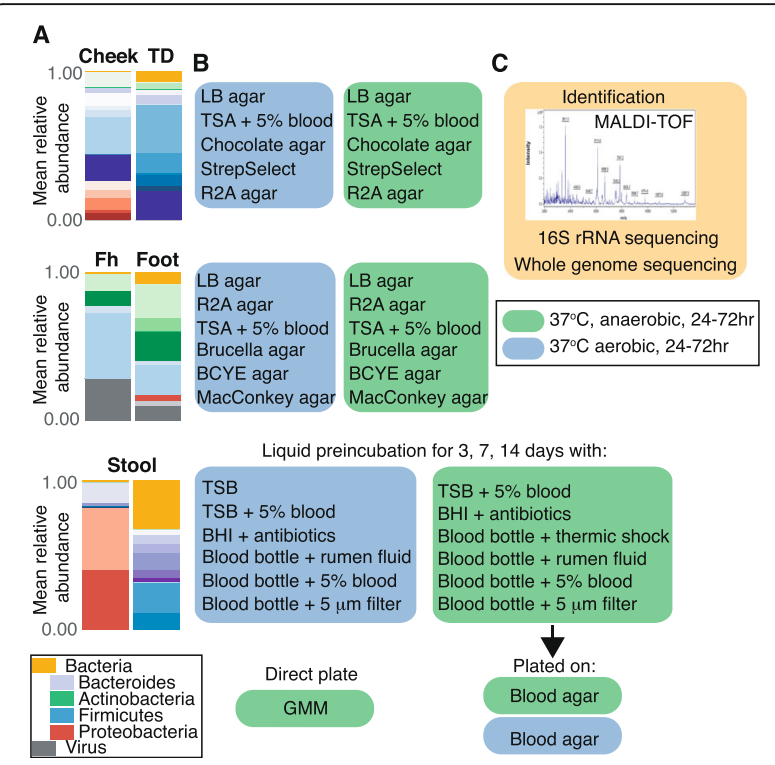

Fig. 1 Culturomics pipeline. A) Metagenomic data were generated for each oral (TD: tongue dorsum), skin (Fh: forehead), or stool sample. Example (non-representative) relative abundance plots of major species are shown with colors corresponding to phylum as shown in the legend. Samples were then B) diluted and cultivated in a defined set of anaerobic (green boxes) and aerobic (blue boxes) cultivation conditions for oral, skin, and stool. After a defined period of incubation, individual colonies were picked, subcultured for purity, then C) identified using MALDI-TOF, 16 S rRNA gene sequencing, or whole genome sequencing

17 genera (53 species) in the mouth, and 41 genera (97 species) from stool from 600, 1155, and 1451 isolates tested, respectively (Table 2, Table S3, specieslevel in Table S4). Some bacteria were ubiquitous (isolated from nearly every condition, e.g., Staphylococcus in skin, Streptococcus in oral, Enterococcus and Escherichia in stool, Table S3, species in Table S4). Other microbes had more restricted recovery; for example, Bifidobacterium sp. were only isolated at early timepoints ( $<3$ days, Table S3, Table S4), while recovery of Anaerococcus sp. was achieved only at later timepoints $(>14 \mathrm{~d})$, though we note that these conclusions are impacted by colony sampling and is meant to provide rules of thumb.

We then sought to examine the degree to which these isolates represented the predicted composition from metagenomic data. At the genus level (to account for MALDI-TOF and metagenomic classification accuracy at the species level), we observed an overlap of genera cultivated vs. sequenced of $44.4 \pm 7.7 \%$ (cheek, mean \pm standard deviation), $27.9 \pm 5.4 \%$ (tongue dorsum), $24.1 \pm$ $12.4 \%$ (forehead), $24.1 \pm 18.0 \%$ (toeweb), and $18.1 \pm 2.3 \%$ (stool, Fig. 2B-C). These represented a high proportion of the community composition reconstructed with metagenomic sequencing (Fig. 2D); $87.7 \pm 7.5 \%, 66.2 \pm 13.8 \%$,
$95.8 \pm 3.7 \%, 92.6 \pm 16.1 \%$, and $67.9 \pm 21.7 \%$, respectively, suggesting that our methods captured the majority of the abundant genera irrespective of body site and that many of the missing genera were potentially low abundance microbes. Correlation analysis between relative abundance and frequency of cultivation (number of times an isolate was identified), by body site, showed a range of associations (species- and genus-level Spearman's rho and $p$-values reported in Table S5, example scatterplots in Fig. S2). Some species showed a positive correlation (e.g., Staphylococcus sp. lugdunensis, pettenkoferi, warneri, although at the genus level Staphylococcus was not positively associated), and in some cases a significant negative association (e.g., E. coli), reflecting low relative abundance but high frequency of cultivation.

We then examined if, and what genera were preferentially cultivated and found there were genera that were identified only by metagenomic sequencing but also only by cultivation (Table 2, Fig. 2B-C, Table S5 for species and genus-level). Metagenomics, as expected, definitively recovered a larger number of total microbes that were not captured by culturomics (Fig. 2B). Prevalent metagenomic genera that were not cultured were primarily anaerobes from the gut and oral cavity, including Capnocytophaga (phylum Bacteroidetes, found in 9 samples), Porphyromonas [7], Alloprevotella [7], Actinobacillus (phylum Proteobacteria, 7), and Leptotrichia (phylum Fusobacteria, 7). We were also surprised that we consistently identified species that were not captured by metagenomics. Interestingly, Enterococcus (phylum Firmicutes) was never identified by sequencing, but fairly extensively cultured as several species (E. avium, faecalis, faecium across six samples). Similarly, Bacillus species (B. circulans, pumilus, subtilis) were only identified in one metagenomic sample (as Bacillus amyloliquefaciens) compared to five samples for culturomics. Even wellstudied bacteria like staphylococci could be cultivated from a sample more frequently than detected by metagenomic data; $8 / 17$ times it was only detected by cultivation. Potential explanations for these observations could include: 1) A species is easily cultivatable (e.g., staphylococci, Enterococcus) but low abundance, resulting in insufficient reads that can be classified to a species by algorithms such as Metaphlan2 [49], which map reads to a limited set of species-specific marker genes, 2) incomplete or few reference genomes are available for that genus to enable its classification (e.g., Bacillus sp., which have relatively fewer reference genomes compared to Enterococcus with hundreds of deposited reference genomes). This would also result in a limited ability of Metaphlan2-like algorithms that identify clade-specific marker genes to identify robust discriminatory features. Here, mapping of metagenomic data selectively to 


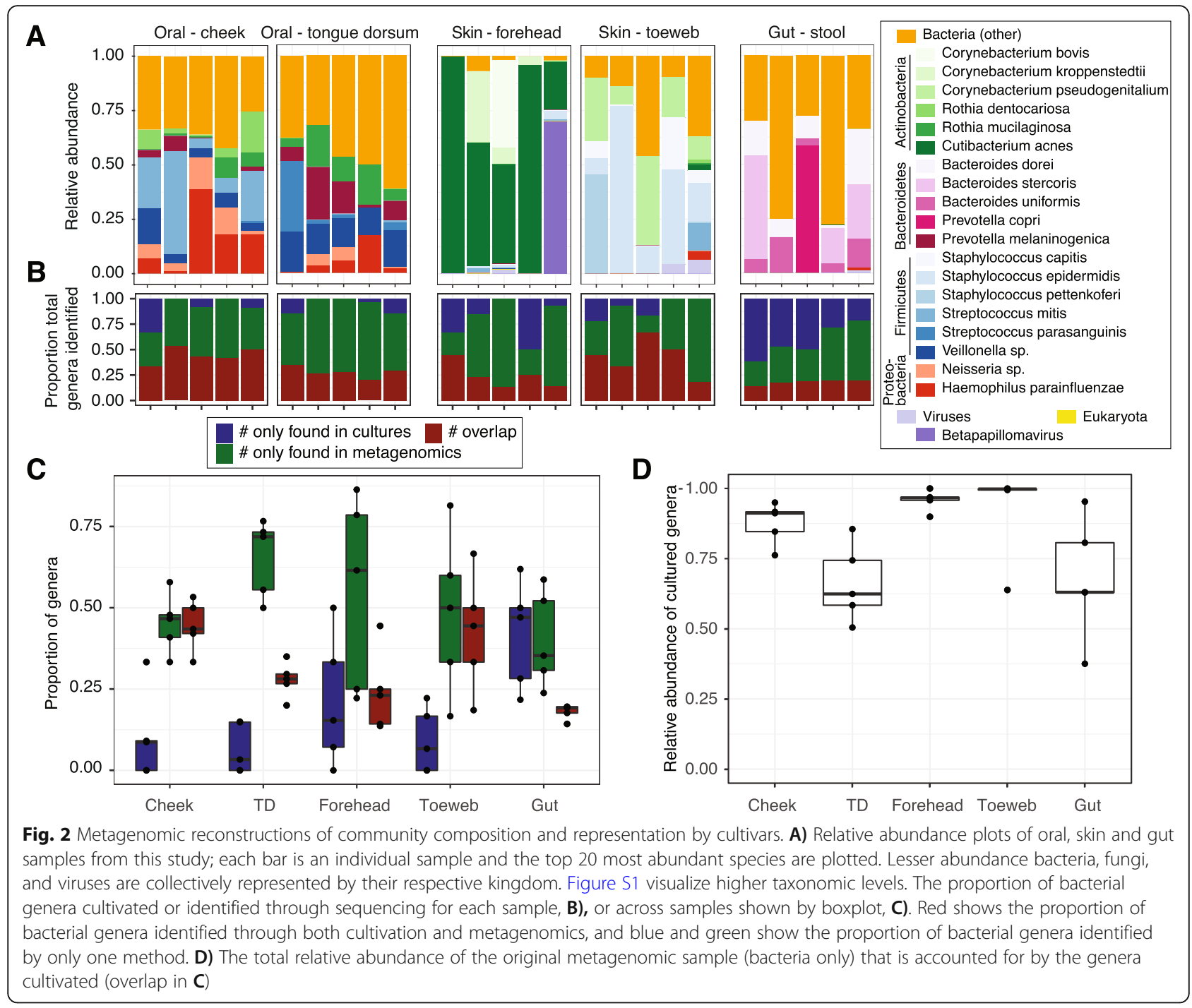

Enterococcus reference genomes confirmed a very low mapping rate and thus relative abundance in the sample.

\section{Strain identification}

An emerging frontier of metagenomic discovery is the understanding of strain biology, as microbial diversity is ultimately manifested at this finest taxonomic resolution where individual strains of a microbial species can exhibit widely diverse phenotypes. As our methods primarily recover dominant microbes (e.g., staphylococci, $E$. coli), it is particularly effective for investigating strain variation between individuals and cohorts. Further, extensive strain variation can exist not only between, but as we have shown, within individuals [7]. This phenomenon has major implications for disease severity [7]. It is thus valuable to be able to rapidly differentiate strains to understand strain diversity, to identify diseasecausing strains, and to prioritize strains for phenotyping.
Different methods with widely differing resolution have been developed for strain typing primarily for clinical use, perhaps most commonly multi-locus sequence typing (MLST) [50], which sequences polymorphisms in highly conserved genes to bin strains into sequence 'types'. The gold standard is whole genome sequencing, but despite extraordinary technical advances, it remains relatively costly and slow to perform and analyze on large scales. Other nucleic acid-based approaches, such as RepPCR, leverage strain-specific differences in repetitive regions to discriminate (but not identify) strains, but can be time consuming to deploy with multiple rounds of PCR, electrophoresis, and interpretation [51, 52]. FT-IR's promise is in its low-cost and rapid generation of discriminatory biochemical fingerprints primarily based on cell surface macromolecules, e.g., lipids, proteins, and carbohydrates. It has been used in examining clonal outbreaks of varying origin, although overall it remains less frequently used despite its lengthy technical history (reviewed in [53]), 
Table 2 Genera uniquely detected by culturomics, metagenomics, and overlap

\begin{tabular}{|c|c|c|c|c|c|}
\hline Phylum & Genus & \# samples cultured only & \# samples metagenomic only & \# overlaps & \# total samples \\
\hline Actinobacteria & Corynebacterium & 5 & 2 & 12 & 19 \\
\hline Firmicutes & Streptococcus & 1 & 7 & 11 & 19 \\
\hline Firmicutes & Veillonella & 0 & 7 & 10 & 17 \\
\hline Proteobacteria & Neisseria & 0 & 4 & 10 & 14 \\
\hline Actinobacteria & Actinomyces & 2 & 3 & 10 & 15 \\
\hline Actinobacteria & Rothia & 0 & 6 & 9 & 15 \\
\hline Firmicutes & Staphylococcus & 8 & 0 & 9 & 17 \\
\hline Actinobacteria & Cutibacterium & 1 & 3 & 8 & 12 \\
\hline Proteobacteria & Haemophilus & 0 & 9 & 7 & 16 \\
\hline Firmicutes & Gemella & 0 & 7 & 6 & 13 \\
\hline Firmicutes & Lachnoanaerobaculum & 0 & 5 & 5 & 10 \\
\hline Bacteroidetes & Bacteroides & 0 & 1 & 5 & 6 \\
\hline Bacteroidetes & Parabacteroides & 0 & 0 & 5 & 5 \\
\hline Fusobacteria & Fusobacterium & 1 & 3 & 4 & 8 \\
\hline Firmicutes & Lactobacillus & 2 & 2 & 3 & 7 \\
\hline Actinobacteria & Bifidobacterium & 0 & 1 & 3 & 4 \\
\hline Actinobacteria & Collinsella & 0 & 0 & 3 & 3 \\
\hline Bacteroidetes & Prevotella & 0 & 12 & 2 & 14 \\
\hline Proteobacteria & Aggregatibacter & 0 & 6 & 2 & 8 \\
\hline Firmicutes & Clostridium & 3 & 4 & 2 & 9 \\
\hline Actinobacteria & Micrococcus & 2 & 2 & 2 & 6 \\
\hline Proteobacteria & Escherichia & 3 & 1 & 2 & 6 \\
\hline Actinobacteria & Brevibacterium & 1 & 1 & 2 & 4 \\
\hline Firmicutes & Flavonifractor & 2 & 0 & 2 & 4 \\
\hline Firmicutes & Granulicatella & 0 & 13 & 1 & 14 \\
\hline Actinobacteria & Atopobium & 1 & 5 & 1 & 7 \\
\hline Actinobacteria & Kocuria & 1 & 5 & 1 & 7 \\
\hline Firmicutes & Eubacterium & 1 & 3 & 1 & 5 \\
\hline Proteobacteria & Acinetobacter & 0 & 3 & 1 & 4 \\
\hline Bacteroidetes & Alistipes & 0 & 3 & 1 & 4 \\
\hline Firmicutes & Blautia & 1 & 2 & 1 & 4 \\
\hline Bacteroidetes & Odoribacter & 1 & 2 & 1 & 4 \\
\hline Actinobacteria & Kytococcus & 1 & 1 & 1 & 3 \\
\hline Firmicutes & Bacillus & 5 & 0 & 1 & 6 \\
\hline Actinobacteria & Dermabacter & 2 & 0 & 1 & 3 \\
\hline Bacteroidetes & Capnocytophaga & 0 & 9 & 0 & 9 \\
\hline Proteobacteria & Actinobacillus & 0 & 8 & 0 & 8 \\
\hline Fusobacteria & Leptotrichia & 0 & 7 & 0 & 7 \\
\hline Bacteroidetes & Porphyromonas & 0 & 7 & 0 & 7 \\
\hline Bacteroidetes & Alloprevotella & 0 & 7 & 0 & 7 \\
\hline Firmicutes & Oribacterium & 0 & 6 & 0 & 6 \\
\hline Firmicutes & Stomatobaculum & 0 & 6 & 0 & 6 \\
\hline Firmicutes & Subdoligranulum & 0 & 6 & 0 & 6 \\
\hline Proteobacteria & Campylobacter & 2 & 5 & 0 & 7 \\
\hline
\end{tabular}


Table 2 Genera uniquely detected by culturomics, metagenomics, and overlap (Continued)

\begin{tabular}{|c|c|c|c|c|c|}
\hline Phylum & Genus & \# samples cultured only & \# samples metagenomic only & \# overlaps & \# total samples \\
\hline Firmicutes & Peptostreptococcus & 2 & 5 & 0 & 7 \\
\hline Firmicutes & Megasphaera & 0 & 5 & 0 & 5 \\
\hline Firmicutes & Solobacterium & 0 & 5 & 0 & 5 \\
\hline Firmicutes & Oscillibacter & 0 & 5 & 0 & 5 \\
\hline Proteobacteria & Enhydrobacter & 0 & 4 & 0 & 4 \\
\hline Firmicutes & Abiotrophia & 0 & 4 & 0 & 4 \\
\hline Proteobacteria & Kingella & 0 & 4 & 0 & 4 \\
\hline Firmicutes & Dorea & 0 & 4 & 0 & 4 \\
\hline Proteobacteria & Bilophila & 0 & 4 & 0 & 4 \\
\hline Firmicutes & Faecalibacterium & 0 & 4 & 0 & 4 \\
\hline Firmicutes & Finegoldia & 2 & 3 & 0 & 5 \\
\hline Firmicutes & Selenomonas & 0 & 3 & 0 & 3 \\
\hline Saccharibacteria & Saccharibacteria & 0 & 3 & 0 & 3 \\
\hline Proteobacteria & Parasutterella & 0 & 3 & 0 & 3 \\
\hline Firmicutes & Roseburia & 0 & 3 & 0 & 3 \\
\hline Firmicutes & Anaerococcus & 3 & 2 & 0 & 5 \\
\hline Firmicutes & Ruminococcus & 2 & 2 & 0 & 4 \\
\hline Firmicutes & Parvimonas & 1 & 2 & 0 & 3 \\
\hline Actinobacteria & Eggerthella & 3 & 1 & 0 & 4 \\
\hline Firmicutes & Pediococcus & 2 & 1 & 0 & 3 \\
\hline Firmicutes & Enterococcus & 6 & 0 & 0 & 6 \\
\hline Proteobacteria & Citrobacter & 3 & 0 & 0 & 3 \\
\hline Actinobacteria & Dietzia & 3 & 0 & 0 & 3 \\
\hline Firmicutes & Lysinibacillus & 3 & 0 & 0 & 3 \\
\hline
\end{tabular}

likely because of incomplete understanding of the link between genetic diversity and cell surface macromolecular diversity.

Here, we evaluated the ability of the Bruker IR Biotyper to rapidly differentiate genetically diverse strains from phylogenetically diverse species, selected as common species of interest in the skin, oral, or gut microbiota. In addition to cultivars obtained in our study, we included additional publicly available, fully sequenced isolates to provide additional genetic diversity. Finally, by way of benchmark, we sequenced, or obtained from public repositories, the genomes of these strains to determine genetic relatedness (Table S1), although we recognize that the cell surface macromolecules are encoded and modified by numerous genetic pathways and environmental conditions, like length of growth time and composition of growth media, and are likely difficult to translate to genetic distance, as previously noted [53].

We investigated IR's ability to differentiate genetically diverse strains of $S$. aureus, $S$. epidermidis, $C$. acnes as important skin microbes, and E. coli and B. subtilis from the gut. We primarily investigated diverse isolates obtained from different individuals, with the exception of $E$. coli in which we investigated within-individual diversity (or clonality) by typing multiple isolates obtained from 4 individuals, each with at least 3 technical replicates (i.e., multiple 'spots' of the same colony). To identify general concordance between the phylogenetic distance (genomics, Fig. 3A) and biochemical distance (IR) between isolates for each species, we performed an exploratory analysis comparing dendrograms (Fig. 3C) and principal component analysis (Fig. 3B) generated from both datatypes.

Nearly universally, technical replicates were the most similar to each other, irrespective of species and supporting IR's reproducibility. For each species, we give some examples of concordance and discordance of clusters formed by IR vs. genome sequencing (Fig. 3).

1) In most cases, $S$. aureus strains formed distinct clusters; in particular, S. aureus PS187, Newman1, and NR-45944 which were also identified as more distant phylogenetically. However, in cases where overlapping clusters were identified by IR (e.g., 1013.MSR-26 \& ATCC 29213, SA113 \& NRS140, 


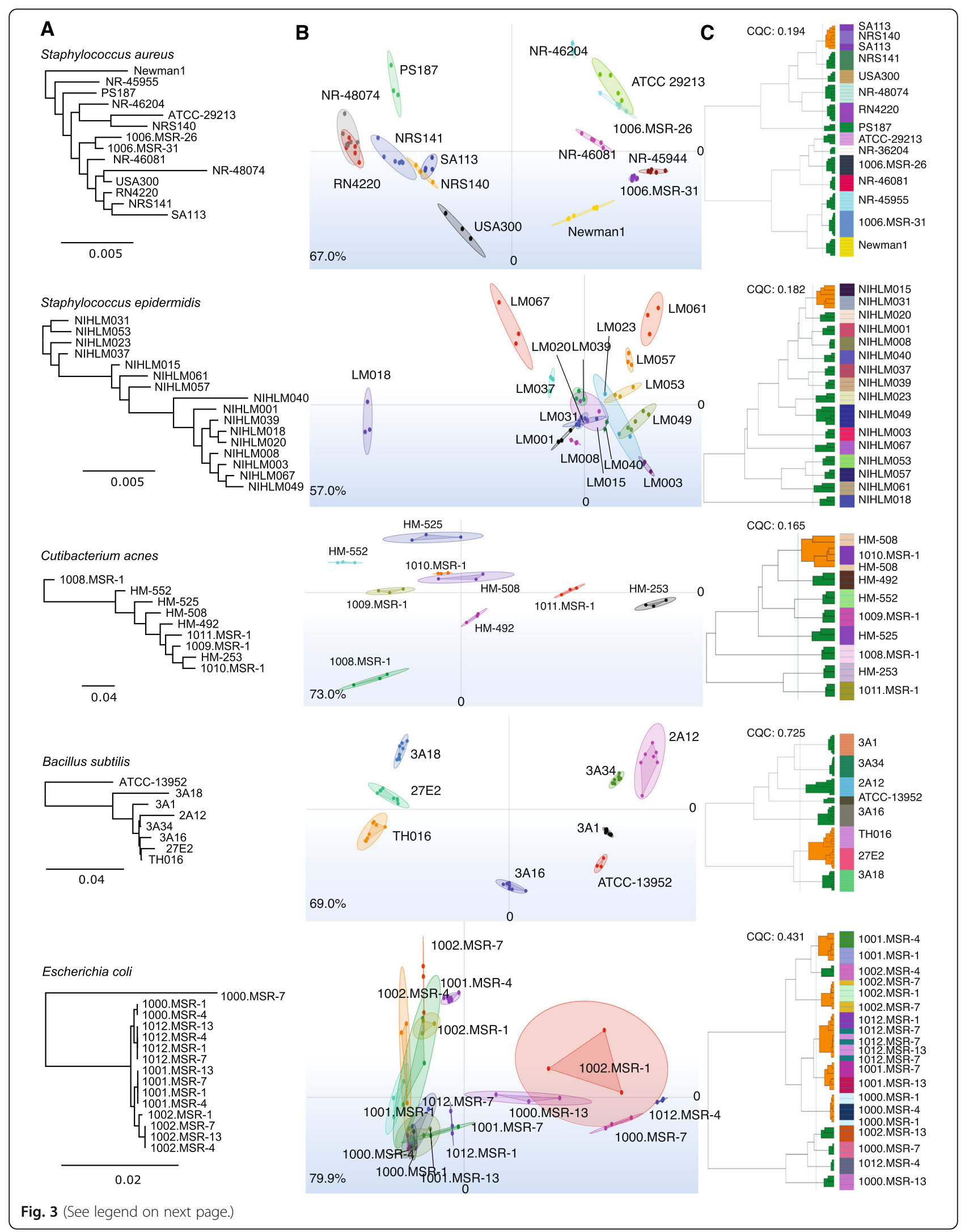


(See figure on previous page.)

Fig. 3 Biotyper IR differentiates genetically distinct strains. A) Phylogenetic trees of strain genomes tested in the Biotyper IR analysis based on alignment of bacterial marker genes. Genetic distance is shown in a dendrogram; genomes used (generated in this study or obtained from public repositories) are in Table S1. B) Principal component analysis (PCA) plot showing clustering of strains for each species, with each color representing a unique isolate and each dot within that color representing the isolate's replicate spectral measurements. Links to the dots showing the variance of the technical replicates; output from IR Biotyper interface. \#\# in lower left corner indicate the sum of variance explained by the first two principal components. C) Dendrogram of isolates based on spectral measurements; output from IR Biotyper interface. Green and orange in dendrogram represent cluster purity as determined by the Bruker IR software, based on technical replicates of strain spectra: green ("GOOD"), orange ("BAD"). Cluster quality criterion (CQC) indicates how well replicate measurements of an isolate cluster with themselves as well as the purity or homogeneity of each cluster

and RN4220 \& NR-48074), most were relatively genetically distinct. In some cases, genetic and IR clusters were recapitulated (NRS141 \& SA113, NR46204 \& ATCC-29213). Surprisingly, 1006.MSR-31 and 1006.MSR-26 were both highly genetically similar (isolated from the same individual) but did not form overlapping clusters.

2) We observed two clades of $S$. epidermidis strains, consistent with our previous large-scale genome analysis [7]. However, these clades were not recapitulated by IR, with the most distinct IR clusters coming from more genetically related strains (NIHL M018, NIHLM067, NIHLM061). Even highly genetically similar strains (e.g., NIHLM018 and NIHL M020), formed distant and distinct clusters.

3) C. acnes strains, each isolated from different individuals, largely formed distinct clusters with the exception of 1008.MSR-1 and HM-508 which shared overlapping clusters, but were genetically distant. HM-253 and 1010.MSR-1 were genetically most similar but were more closely related to other strains by IR spectra.

4) The B. subtilis strains tested had relatively higher genetic distance than the previous species. TH016 and 27E2, which were genetically most similar, formed overlapping IR clusters, but 3A18, which was relatively more distant, was also a near neighbor. ATCC-13952 robustly formed a distinct cluster and was an outlier genetically. In the case of $B$. subtilis, we also tested biological replicates (independent colonies between two separate runs), which yielded highly concordant results with strains from both runs grouping into the same cluster(s) (Fig. S3).

5) The ability to differentiate $E$. coli, where we examined both within- and between-individual variation, differed from the other species tested in its relatively lower clarity in strain differentiation by IR. However, upon closer look, in most cases, strains from the same patient clustered together based on their spectral profile (e.g., 1001.MSR-4 \& 1001.MSR-1, 1002.MSR-4 \& 1002.MSR-1 \& 1002.MSR-7, 1012.MSR-1 \& 1012.MSR-7 \&
1012.MSR-13, 1000.MSR-1 \& 1000.MSR-4). Because $E$. coli within an individual likely derives from a single lineage (like $B$. fragilis in the gut [54]), this underscores a strength of the IR in its ability to discriminate clonal strains from other strains.

A surprising result was 1000.MSR-7, which had been identified as E. coli by MALDI-TOF, and which genetically was relatively distant from the other $E$. coli strains (taxonomic identification based on alignment of a set of core marker genes), including other strains from the same patient, but shared an IR spectral cluster with another patient's strain (1002.MSR-13). Upon closer examination of the whole genome sequence, this strain, which had been identified as E. coli by MALDI-TOF, was classified as Klebsiella pneumoniae, which shares $99.01 \%$ ANI (average nucleotide identity) in the single copy marker genes used for classification. This particular discrepancy is mostly likely attributable to a limited ability of MALDI-TOF to differentiate these species. Anecdotally, we have observed similar results when testing different strains from different species on the IR (data not shown). This reinforces the need for strain isolation coupled with rapid and low-cost approaches that differentiate the strains by either genomic or phenotypic features.

\section{Discussion}

Vis a vis our general cultivation pipelines, we note two general observations. First, we observed a significantly different recovery rate for many microbes. For example, staphylococci in the skin, E. coli and Enterococcus in the gut, and streptococci in the oral cavity are recovered with far greater frequency and repetition than more abundant but more fastidious microbes. Second, we found that most microbes could be recovered on multiple growth conditions, and that there were relatively few media that specifically allow recovery of a desired taxon. For example, selective staphylococcal media (e.g., SaSelect culture plates) is designed for colorimetric differentiation of staphylococci, but frequently recovers $\mathrm{Ba}$ cillus and Micrococcus. Anecdotally, we have explored depletion of such microbes like staphylococci by 
antibiotics, lysostaphin, and crystal violet, which resulted in low-to-moderate depletion, and per the goals of the project at hand, are recommended for further exploration. Overall, we believe that our approach results in broad recovery of abundant bacteria and low-abundance, non-fastidious bacteria, and as such, this general philosophy may be must useful for recovering and rapidly differentiating patient-specific strains of these microbes. For deeper recovery of the microbial diversity in a sample, we thus recommend on a per-application basis, evaluation of targeted approaches for the recovery of desired microbes, either via depletion of abundant microbes, increased number of growth conditions, and potentially most importantly, increased numbers of colonies surveilled. In addition, some microbes are epibionts, requiring co-culture for growth e.g. [25],. In addition, an increasing number of innovative approaches, including engineered antibody capture [25], microfluidics devices that can be placed in the environment [24], 3-D organoids to better recapitulate growth environments, and high throughput content screenings, are being developed to facilitate increased recovery of desired microbes for follow-up experimentation.

For strain identification, our results suggest that IR's ability to differentiate strains is first, likely speciesspecific. This means that different species can have notably different levels of strain-level genetic diversity, i.e., some bacteria have relatively closed vs. open pangenomes, e.g., C. acnes [55] and S. epidermidis [7], respectively. That may differentially affect cell surface macromolecule diversity and hence the IR readout. Second, IR is likely most valuable in differentiating clonal strains from any other non-clonal strain, rather than making a general assessment of genetic divergence and phylogenetic placement. In this way, we found that the IR had particular value to our goals of selecting unique strains from dominant microbes. In cultivating dominant microbes, we typically observe 'frequent fliers', i.e., microbes cultivated repeatedly. However, from MALDITOF it is impossible to determine if these are clonal both within and between individuals. For example, Bacteroides fragilis has been deemed relatively clonal in the gut [54], and thus selecting just one representative isolate per individual might suffice, but for S. epidermidis, which has extensive within-individual strain diversity [7], several representative strains might be chosen. The IR provides sufficient speed and resolution to discriminate clonality, particularly if benchmarked to a reference strain.

It is important to also note limitations of the IR. For example, run-to-run reproducibility in differentiating the same set of strains was strong (Fig. S2), but it is difficult to directly compare between runs, particularly with a large strain set. This is because different sets of strains from different runs are analyzed within-run, rather than benchmarked to an external database. Thus, given the 96-spot format, 30 strains can be typed simultaneously accounting for technical triplicates. Second, as we previously noted, real genetic distance is difficult to deconvolute without an extensive paired comparative genomics approach. Nevertheless, despite these limitations, we believe that this rapid strain differentiation would be useful for selecting a subset of isolates from a set of patient cultivars that minimizes likely phenotypic redundancy.

Finally, we comment on practicalities for deploying these approaches. In mid-2021 costs with intermediate personnel, we estimated the stool pipeline described herein to cost $\sim \$ 450 /$ sample, reflecting the relatively greater reagent costs and extensive person-hours $(\sim 10$ person hours) with the numerous conditions and limited multiplexing (4 samples at a time). Oral and skin sites were significantly lower in both costs and labor at $\sim$ \$125/sample ( 2 person hours/site), though these numbers benefit from scalability as again, we seldom cultivated from a single skin or oral site. However, there is significant value in using MALDI-TOF and IR technologies, as a set of 96 species or 30 strains can be profiled in 30 or $60 \mathrm{~min}$ for $\$ 15$ and $\$ 175$, respectively, at 2021 reagent costs using a 2016 machine. In contrast, $16 \mathrm{~S}$ rRNA sequence classification or whole genome sequencing approximates $\$ 10 /$ sample and $\sim 30-\$ 50$ /genome (extensively multiplexed on Novaseq S4) for reagents alone, respectively, and is at its most rapid, $24 \mathrm{~h}$ hour turnaround time for $16 \mathrm{~S}$ sequencing and $\sim$ a week for a fully analyzed genome sequence.

\section{Conclusions}

Here, we have presented and characterized a facile workflow for cultivation of bacteria from skin, oral, and gut microbiota from genus/species to the strain level with a focus on throughput across many patient samples rather than comprehensive recovery of all the species within a sample. We believe these efforts add to an increasing body of approaches for translating genomics-driven discoveries to microbial mechanism, and highlights the value-add of strain-level analysis to better understand genetic and phenotypic diversity underlying hostmicrobiome interactions.

\section{Methods}

\section{Sample acquisition}

Forehead and toeweb swabs (skin) and inner cheek and dorsum of the tongue (oral) swabs, and stool samples were obtained from our internal repository of human samples (approved by the Jackson Laboratory Institutional Review Board). Altogether in this study, we used 25 different samples obtained from 12 individuals. For skin and oral microbiota, sites were swabbed rigorously using 2 PurFlock Ultra buccal swabs (Puritan ${ }^{\text {Tw }}$ PurFlock 
Ultra, \#22-029-506) for each site for $30 \mathrm{~s}$ before one swab was submerged into a $1.5 \mathrm{~mL}$ Eppendorf tube containing $500 \mu \mathrm{l}$ Reasoner's 2A (R2A) broth culture media (Lab M, LAB203-A) and the other into a microfuge tube containing $350 \mu \mathrm{L}$ Tissue and Cell Lysis buffer (Epicentre, MPY80200) and $100 \mu \mathrm{g} 0.1 \mathrm{~mm}$ zirconia beads (BioSpec Products, 11,079,101) for whole-genome shotgun metagenomic sequencing. Stool was self-collected at home by volunteers using a BioCollector fecal collection kit (The BioCollective, Denver, CO) according to manufacturer instructions. The volunteers also added a portion of the stool sample to an OMNIgene.GUT tube (DNA Genotek, OMR-200) following manufacturer instructions for preservation for sequencing prior to sending the sample in a provided Styrofoam container with a cold pack. Upon receipt, stool and OMNIgene samples were immediately aliquoted and frozen at $-80^{\circ} \mathrm{C}$ for storage. Stool samples were homogenized inside the kit sample transport bag manually by massage and compression until combined (5-10 s), then roughly $1 \mathrm{~mL}$ was aliquoted into microfuge tubes. Prior to aliquoting, OMNIgene stool samples were homogenized by vortexing (using the metal bead inside the OMNIgene tube), then divided into 2 microfuge tubes, one with $100 \mu \mathrm{L}$ aliquot and one with $1 \mathrm{~mL}$.

\section{Cultivation conditions (Table 1) Cultivation approach}

Skin and oral. The $1.5 \mathrm{~mL}$ Eppendorf tube containing R2A and swab was thoroughly vortexed, and then diluted 1:100 and 1:1000 in R2A, to increase the chance of recovering single colonies. $50 \mu \mathrm{L}$ from each dilution was then spread on half of an agar plate for each cultivation condition using sterile glass beads (Fisher Scientific, 50-444635) or a sterile spread tool (Thomas Scientific, 229,616).

Stool. Approximately $5 \mathrm{~g}$ of stool sample was thawed and added to a $50 \mathrm{~mL}$ conical tube containing either 15 $\mathrm{mL}$ PBS (for aerobic culture) or $15 \mathrm{~mL}$ deoxygenated PBS with 0.1\% L-cysteine (Sigma-Aldrich, 168,149) (for anaerobic culture), then vortexed well for $5 \mathrm{~min}$ and left to settle for $15 \mathrm{~min}$. For the anaerobic direct plating condition, serial PBS/L-cysteine dilutions of 1:10,000 and 1: 100,000 of each sample were plated on gut microbiota medium (GMM) plates [3] and left to incubate at $37{ }^{\circ} \mathrm{C}$ degrees in the anaerobic chamber for 48-72 h until colony formation was observed. Blood culture-assisted cultivation was utilized to select for the growth of underrepresented and slow-growing species, the PBS/ stool mixture was added to a variety of culture conditions and incubated for $3,7,14$, and 28 days prior to being diluted in PBS/L-cysteine and plated on blood agar plates (Table 1 and Fig. 1). Plates were incubated in the atmosphere and temperature of the original culture for 24-72 h until colony formation was observed.

\section{Media and supplemental items}

For liquid and agar cultivation as detailed in Table 1, the following mediums were sourced from Fisher Scientific: Luria Broth (LB) agar (BP1425500), R2A agar (R454372), Trypitic Soy Broth (TSB) (DF0370-17-3), Brain Heart Infusion (BHI) (DF0037-17-8), Chocolate agar (B21169X), Buffered Charcoal Yeast Extract (BYCE) agar (B21808), Tryptic Soy Agar (TSA) with 5\% sheep blood agar (B21261X). Brucella agar was sourced from Anaerobe Systems (AS-141), while MacConkey and Selective Strep agars were purchased from Hardy Diagnostics (GA35 and A70 respectively). The supplemental additions included sheep's blood (Fisher Scientific, R54008), rumen fluid (Fisher Scientific, NC9821770), vancomycin (Sigma-Aldrich, V1139) and colistin (Sigma-Aldrich, C4461). Aerobic and anaerobic blood bottles were purchased from Fisher Scientific (23-032512 and 23032513 respectively). The $5 \mu \mathrm{m}$ filters were purchased from Fisher Scientific (SLSV025LS).

\section{Environments}

The anaerobic atmosphere consisted of 5\% hydrogen, 5\% carbon dioxide, 90\% nitrogen (Airgas, Z03NI9022000008). Aerobic cultures were conducted in ambient atmosphere.

\section{Isolate identification \\ MALDI-TOF}

We used matrix assisted laser desorption ionization-time of flight (MALDI-TOF, Bruker Daltonics, Germany) mass spectrometry to identify isolates. Ten to twelve single colonies from each cultivation condition were picked and replated onto a new blood agar plate (Fisher Scientific, $\mathrm{B} 21261 \mathrm{X})$, then grown for $24-48 \mathrm{~h}$ to generate sufficient material for identification and archiving. Using a sterile transfer device (Puritan, 25-28,107), bacteria were directly transferred to a MALDI target spot. We then used the 'extended direct method' for sample preparation, in which $70 \%$ formic acid (Sigma-Aldrich, 5,438,040,100) is used to solubilize the bacterial cell wall prior to addition of matrix (Bruker Matrix HCCA, 14932). One spot on the target was reserved for the bacterial test standard (Bruker, 8,255,343) for calibration. Mass spectrometry analysis was performed using Flex Control 3.4 software (Bruker Daltonics, Germany). Identification score values below 1.60 were considered failed. Colonies not recognized by MALDI-TOF were processed using the 'protein extraction' method, and failing that by $16 \mathrm{~S}$ rRNA gene sequencing as detailed below.

\section{Protein extraction}

Bacteria failing identification by the extended direct method were then extracted. $10 \mathrm{mg}$ of biological material (generous scoop of a $1 \mu \mathrm{L}$ inoculation loop) was thoroughly suspended in $300 \mu \mathrm{L}$ of High-Performance Liquid Chromatography water (Sigma-Aldrich, 900,682) then 
thoroughly mixed with $900 \mu \mathrm{L} 100 \%$ ethanol in a $1.5 \mathrm{~mL}$ Eppendorf tube. The tube was then centrifuged at $13,000 \mathrm{rpm}$ for $2 \mathrm{~min}$ and the supernatant decanted. The tube was centrifuged again, and remaining ethanol was removed with a small pipet or allowed to air dry at room temperature. $10 \mu \mathrm{L}$ of $70 \%$ formic acid was thoroughly mixed to the pellet by pipetting, followed by $10 \mu \mathrm{L}$ acetonitrile (Sigma-Aldrich, 900,667), then centrifuged for another $2 \mathrm{~min} .1 \mu \mathrm{L}$ of supernatant was used on each MALDI target spot, dried, and overlayed with $1 \mu \mathrm{L}$ of matrix. Mass spectrometry analysis was performed as above.

\section{5 rRNA sequencing}

Bacteria failing identification by mass spectrometry and protein extraction were identified by $16 \mathrm{~S}$ rRNA gene sequencing. Alkaline lysis was used to generate microbial DNA for PCR using universal primers 8F and 1391R (Turner, 1999). Sanger sequencing (GeneWiz, Inc) results were analyzed using BLAST (blast.ncbi.nlm.nih. gov). An identity score of $99 \%$ or higher was the threshold used for accurate species identification.

For archiving, the recovered bacteria were grown in TSB supplemented with $0.1 \mathrm{mg} / \mathrm{L}$ Vitamin K (Sigma-Aldrich, 95,271) and $5 \mathrm{mg} / \mathrm{L}$ hemin (Sigma-AldrichH9039) $24-48 \mathrm{~h}$ in 96 -well plates at $37^{\circ} \mathrm{C}$ in the appropriate atmosphere, then stored in $20 \%$ glycerol (Fisher Scientific, BP229-1) at $-80^{\circ} \mathrm{C}$.

\section{Whole genome sequencing}

Rapid DNA extraction from S. epidermidis isolates was adapted from Köser et al. (2014) and were performed as in Zhou et al. (2020) [7]. Briefly, $1 \mathrm{~mL}$ of overnight culture was centrifuged at $20,000 \mathrm{x}$ g for $1 \mathrm{~min}$. The bacterial pellet was then resuspended in $100 \mu \mathrm{L}$ of $1 \mathrm{X}$ TEbuffer and transferred to a $2 \mathrm{~mL}$ bead beating tube with 100-125 $\mu \mathrm{L} 0.5 \mathrm{~mm}$ diameter glass beads (BioSpec Products, NC0417355) for homogenization. An extra $100 \mu \mathrm{L}$ of $1 \mathrm{X}$ TE-buffer was added to the tube, which was then vortexed for $30 \mathrm{~s}$ at $3000 \mathrm{rpms}$ using a vortex adaptor (Mo Bio Laboratories). Tubes were then centrifuged at $13,000 \times \mathrm{g}$ for $5 \mathrm{~min}$ to pellet the majority of cellular debris. The majority of the supernatant, taking care to not disturb the pellet, was then transferred to a fresh tube for quantitation of DNA using a Qubit 2.0 Fluorometer (Thermo Fisher Scientific). Supernatant was then diluted to $160 \mathrm{pg} / \mu \mathrm{l}$. To make Nextera XT libraries, we used the Illumina standardized protocol (Nextera XT DNA sample preparation kit, Illumina Inc., FC-131-1096), creating dual indexed paired-end libraries. We adapted and miniaturized this protocol by taking all reagents in 1/4th amount and using $200 \mathrm{pg}$ of DNA for each reaction, to generate an average insert size of $400 \mathrm{bp}$. Tagmentation and PCR reactions were carried out according to manufacturer's instructions, and the resultant libraries were sequenced with 2 X150bp paired end reads on an Illumina HiSeq2500 targeting $\sim 5$ million paired-end reads per sample.

\section{Metagenomic sequencing Skin and Oral}

We used our established protocol for metagenomic extractions [7]. DNA from swabs stored in lysis buffer and glass beads was extracted using the GenElute Bacterial DNA Isolation kit (Sigma-Aldrich, NA2110-1KT) with the following modifications: $5 \mu \mathrm{L}$ of Lysozyme $(10 \mathrm{mg} /$ $\mathrm{mL}$, Sigma-Aldrich, L6876), $1 \mu \mathrm{L}$ Lysostaphin (5000 U/ $\mathrm{mL}$, Sigma-Aldrich, L9043) and $1 \mu \mathrm{L}$ Mutanolysin (5000 U/mL, Sigma-Aldrich, M9901) were added to each sample, allowed to digest at $37^{\circ} \mathrm{C}$ for $30 \mathrm{~min}$. Then, samples were homogenized by bead-beating in a TissueLyser II (QIAGEN) for $2 \times 3 \mathrm{~min}$ at $30 \mathrm{~Hz} .5 \mu \mathrm{L}$ of proteinase $\mathrm{K}$ $(20 \mathrm{mg} / \mathrm{mL}$, Sigma-Aldrich) and $300 \mu \mathrm{L}$ of Solution C was then added and samples incubated at $55^{\circ} \mathrm{C}$ for 30 min. $300 \mu \mathrm{L}$ of $100 \%$ ethanol was used to precipitate the samples. Each sample was centrifuged for $1 \mathrm{~min}$ at $15000 \mathrm{x} \mathrm{g}$ prior to loading onto the GenElute column. Negative (environmental) controls and positive (in-house mock community of 26 unique bacterial species) controls were extracted and sequenced with each extraction and library preparation batch to ensure sample integrity. Subsequent steps were executed according to manufacturer instructions.

\section{Stool}

Approximately $50 \mu \mathrm{L}$ of thawed OMNIgene preserved stool sample was added to a microfuge tube containing $350 \mu \mathrm{L}$ Tissue and Cell lysis buffer and $100 \mu \mathrm{g} 0.1 \mathrm{~mm}$ zirconia beads. Metagenomic DNA was extracted using the QiaAmp 96 DNA QiaCube HT kit (Qiagen, 5331) with the following modifications: each sample was digested with $5 \mu \mathrm{L}$ of Lysozyme $(10 \mathrm{mg} / \mathrm{mL}$, SigmaAldrich, L6876), $1 \mu \mathrm{L}$ Lysostaphin $(5000 \mathrm{U} / \mathrm{mL}$, SigmaAldrich, L9043) and $1 \mu \mathrm{L}$ oh Mutanolysin $(5000 \mathrm{U} / \mathrm{mL}$, Sigma-Aldrich, M9901) were added to each sample to digest at $37^{\circ} \mathrm{C}$ for $30 \mathrm{~min}$ prior to the bead-beating in the in the TissueLyser II (Qiagen) for $2 \times 3 \mathrm{~min}$ at $30 \mathrm{~Hz}$. Each sample was centrifuged for $1 \mathrm{~min}$ at $15000 \mathrm{x} \mathrm{g}$ prior to loading $200 \mu \mathrm{l}$ into an S-block (Qiagen, 19,585) Negative (environmental) controls and positive (in-house mock community of 26 unique species) controls were extracted and sequenced with each extraction and library preparation batch to ensure sample integrity.

Sequencing adapters and low-quality bases were removed from the metagenomic reads using scythe (v0.994) [56] and sickle (v1.33) [57], respectively, with default parameters, as we have previously performed [7]. Host reads were removed by mapping all sequencing reads to the 
hg19 human reference genome using Bowtie2 (v2.3.1) [58], with "very-sensitive" parameters. Non-human reads (i.e., microbial reads) were used to estimate the relative abundance profiles of the microbial species in the samples using MetaPhlAn2 [49] (database downloaded 3/2020).

To identify Enterococcus species in metagenomic samples, stool metagenomes were mapped directly to reference genomes of $E$. durans (strain 8 L1-82), E. avium (ATCC 14025), E. faecalis (strain 39EA1) and E. faecium (ATCC $8459=$ NRRL B-2354) using bowtie2 (version 2.4.1, -very sensitive mode) [58] extracting mapped reads using samtools (version 1.10 [59]) and then blasting to the respective species using standard parameters. Percent sequence identity was taken from the BLAST [60] results. The same pipeline was successfully applied for $C$. acnes and the skin samples as positive control.

\section{Strain typing with Biotyper IR}

The Bruker IR Biotyper Fourier Transform Infrared (FTIR) Spectroscopy system (Bruker Daltonics, Germany) was used to evaluate strain differences between isolates of a given species. The IR Biotyper analyzes the spectra of peaks corresponding to cell surface glycoproteins and uses hierarchical clustering to establish relationships between strains. Strains were grown from single colonies to a state of confluent growth on tryptic soy agar (TSA) plates. An overloaded $1 \mu \mathrm{L}$ inoculating loop of cell material was resuspended in $50 \mu \mathrm{L}$ of $70 \%$ ethanol (SigmaAldrich) in a $1.5 \mathrm{~mL}$ Bruker suspension vial with inert metal cylinders, and vortexed to homogeneity. $50 \mu \mathrm{L}$ of deionized water was added to the tube, and again vortexed. $15 \mu \mathrm{L}$ of each isolate suspension was pipetted onto 4 spots of a silicon microtiter plate along with 2 spots each of Bruker Infrared Test Standards 1 and 2 (Bruker, 8,255,343). The plate was allowed to dry in a $37^{\circ} \mathrm{C}$ incubator, then loaded into the IR Biotyper for analysis. Spectra were processed by the IR Biotyper software in the 1300-800 cm-1 wavelength, corresponding to the carbohydrate region. Each spectra was comprised of 521 different datapoints. For exploratory analysis to assess similarity of spectra, we used the default IR Biotyper software settings to generate principal components analysis (PCA) plots and dendograms via hierarchical clustering using Euclidean distance to generate distance matrices.

\section{Comparative genomics of microbial genomes}

WGS reads from isolate genomes (see "Whole genome sequencing") were quality-filtered, trimmed, and assembled as described previously [7]. Briefly, sequencing adapters and low quality bases were removed from the sequencing reads using scythe (v0.994) [56] and sickle (v1.33) [57], respectively, with default parameters. Filtered sequencing reads were then assembled using
SPAdes (v3.7.1) [61], with default parameters. The resulting draft genomes, as well as publicly available genomes (Table S1) were analyzed using the classify workflow (with default parameters) of GTDB-Tk (v1.0.2, reference database version r89) [62]. Based on the bacterial marker gene alignment generated by GTDB-Tk, a phylogenetic tree was inferred using FastTree (v.2.1.11) [63] with default parameters and visualized using Figtree (v1.4.4) [64].

\section{Additional statistical analyses}

The frequency of cultivation was computed by counting the number of isolates identified under a given condition. For example, the frequency of the genus Staphylococcus for skin cultivation was the number count of all Staphylococcus species in that skin site, irrespective of the growth condition. Spearman correlations were computed in $\mathrm{R}$ with the base cor.test function. The rho value and the approximate $\mathrm{p}$ - values are shown in Table S5, example scatter plots in Fig. S2. Note here that because of modest sample size and sparse matrix, i.e., multiple zeros in our data (inherent to metagenomic analysis), $\mathrm{R}$ gives the warning 'Cannot compute exact p-value with ties'.

\section{Data deposition}

Strains generated herein are available upon reasonable request. Genomes and metagenomic data are deposited in the Short Read Archive (SRA) under Bioproject PRJNA740337.

\section{Abbreviations}

BCYE: Buffered charcoal yeast extract; BHI: Brain heart infusion; Fh: Forehead; rRNA: 165 ribosomal RNA; C.: Cutibacterium; CQC: Cluster quality criterion; E.: Escherichia; FT: Fourier Transform; IR: Infrared; GMM: Gut microbiota medium; IRB: Institutional Review Board; MALDI-TOF: Matrix assisted laser desorption ionization-time of flight; MLST: Multi-locus sequence typing; PCA: Principal component analysis; S.: Staphylococcus; sp.: Species; TD: Tongue dorsum; TSB: Tryptic soy broth; TSA: Tryptic soy agar; R2A: Reasoner's 2A

\section{Supplementary Information}

The online version contains supplementary material available at https://doi. org/10.1186/s12866-021-02314-y.

Additional file $\mathbf{1}$ Table S1. Metagenomic read count statistics and genomes used in the study. Table S2. Relative abundances of bacteria, fungi, and viruses in metagenomic data. Table S3. Breakdown of genera recovered for each cultivation condition, by body site and timepoint.

Table S4. Breakdown of species recovered for each cultivation condition, by body site and timepoint. Table S5. Spearman correlation between relative abundance in metagenomic data vs. frequency of isolation, genus- and species-level.

Additional file 2 Fig. S1. Relative abundance plots of oral, skin, and gut samples by genus, family, order, class, phylum. Each bar is an individual sample and the top 20 most abundant taxonomic features are plotted.

Additional file $\mathbf{3}$ Fig. S2. Example scatterplots underlying correlation analysis between frequency of species/genera cultivated and relative 
abundance by metagenomic analysis. Correlation of Staphylococcus at $\mathbf{A}$ ) genus- and B) species-level.

Additional file 4 Fig. S3. Run-run reproducibility of Biotyper IR. For two individual runs of $B$. subtilis strains, shown are: A) PCA plot showing clustering of strains for each species, with each color representing a unique isolate and each dot within that color representing the isolate's replicate spectral measurements. Links to the dots showing the variance of the technical replicates; output from IR Biotyper interface. B) dendrogram of isolates based on spectral measurements; output from IR Biotyper interface. Green and orange in dendrogram represent cluster purity as determined by the Bruker IR software, based on technical replicates of strain spectra: green ("GOOD"). Cluster quality criterion (CQC) indicates how well replicate measurements of an isolate cluster with themselves as well as the purity or homogeneity of each cluster.

\section{Acknowledgements}

We thank the Oh lab for critical feedback and the Microbial Genomics Services and Genome Technologies Cores at the Jackson Laboratory for technical assistance. We also thank Michael Santino from Bruker for critical reading of the manuscript.

\section{Authors' contributions}

Conceptualization: JO. Methodology: EF, VP, AH, AP. Analysis: EF, VP, AH, WZ, RH, AYV, YON, JD, RB, AP, ZES, RX, JO. Patient recruitment, evaluation, and sample collection: LB, SDV. Writing: JO, EF, ZES, AH. Resources: JO. All authors have read and approved the manuscript.

\section{Funding}

This project is supported by the National Institutes of Health: (DP2 GM126893-01 (conceptualization (C), study and collection (S), analysis and data interpretation (ADW)), K22 Al119231-01 (C), 1U54NS105539 (C, S, ADW), 1 U19 Al142733 (C, S, ADW), 1 R21 AR075174 (C, S, ADW), R56 AG060746 (C, $S, A D W)$ ), the American Cancer Society (C), the Leo Foundation (C, S, ADW), and the Mackenzie Foundation (C, S, ADW).

\section{Availability of data and materials}

Strains derived are available upon reasonable request, and microbial genomes and metagenomic data is available on the Short Read Archive (SRA) under Bioproject PRJNA740337.

\section{Declarations}

\section{Ethics approval and consent to participate}

The biorepository used for samples in this study was approved by the Jackson Laboratory Institutional Review Board, and all individuals were fully consented, with written assent.

\section{Consent for publication}

Not applicable.

\section{Competing interests}

Dr. Suzanne D. Vernon is is affiliated and has a financial interest with The BioCollective, a company that provided the BioCollector, the collection kit used for at home stool collection discussed in this manuscript. No other authors have competing interests.

\section{Author details}

'The Jackson Laboratory, 10 Discovery Drive, Farmington, CT 860-837-2014, USA. ${ }^{2}$ The University of Connecticut Health Center, Farmington, CT, USA. ${ }^{3}$ Bateman Horne Center, Salt Lake City, UT 84102, USA.

Received: 15 March 2021 Accepted: 7 September 2021

Published online: 14 October 2021

\section{References}

1. Proctor LM, Creasy HH, Fettweis JM, Lloyd-Price J, Mahurkar A, Zhou W, et al. The integrative HMP (iHMP) research network consortium. The Integrative Human Microbiome Project Nature. 2019;569(7758):641-8. https://doi.org/10.1038/s41586-019-1238-8.
2. Faith JJ, Ahern PP, Ridaura VK, Cheng J, Gordon Jl. Identifying Gut MicrobeHost Phenotype Relationships Using Combinatorial Communities in Gnotobiotic Mice. Sci Transl Med. 2014;6(220):220ra11. PMID: 24452263

3. Goodman AL, Kallstrom G, Faith JJ, Reyes A, Moore A, Dantas G, Gordon I. Extensive personal human gut microbiota culture collections characterized and manipulated in gnotobiotic mice. Proc Natl Acad Sci 2011;108(15): 6252-6257. PMID: 21436049, DOl: https://doi.org/10.1073/pnas.1102938108

4. Pasolli E, Asnicar F, Manara S, Zolfo M, Karcher N, Armanini F, Beghini F, Manghi P, Tett A, Ghensi P, Collado MC, Rice BL, DuLong C, Morgan XC, Golden CD, Quince C, Huttenhower C, Segata N. Extensive Unexplored Human Microbiome Diversity Revealed by Over 150,000 Genomes from Metagenomes Spanning Age, Geography, and Lifestyle. Cell [Internet]. 2019 17 [cited 2019 Jan 17];0(0). Available from: https://www.cell.com/cell/abstra ct/S0092-8674(19)30001-7

5. Nayfach S, Pollard KS. Toward accurate and quantitative comparative Metagenomics. Cell. 2016;166(5):1103-1116. PMID: 27565341, DOI: https:// doi.org/10.1016/j.cell.2016.08.007

6. Nayfach S, Shi ZJ, Seshadri R, Pollard KS, Kyrpides NC. New insights from uncultivated genomes of the global human gut microbiome. Nature. 2019; 568(7753):505-510. PMCID: PMC6784871.

7. Zhou W, Spoto M, Hardy R, Guan C, Fleming E, Larson PJ, et al. Host-Specific Evolutionary and Transmission Dynamics Shape the Functional Diversification of Staphylococcus epidermidis in Human Skin. Cell. 2020; 180(3):454-470.e18

8. Leimbach A, Hacker J, Dobrindt U. E. coli as an all-rounder: the thin line between commensalism and pathogenicity. Curr Top Microbiol Immunol 2013;358:3-32. PMID: 23340801, DOl: https://doi.org/10.1007/82_2012_303

9. Quainoo S, Coolen JPM, van Hijum SAFT, Huynen MA, Melchers WJG, van Schaik W, Wertheim HFL. Whole-Genome Sequencing of Bacterial Pathogens: the Future of Nosocomial Outbreak Analysis. Clin Microbiol Rev. 2017;30(4):1015-1063. PMCID: PMC5608882.

10. Linehan JL, Harrison OJ, Han S-J, Byrd AL, Vujkovic-Cvijin I, Villarino AV, Sen SK, Shaik J, Smelkinson M, Tamoutounour S, Collins N, Bouladoux N, Dzutsev A, Rosshart SP, Arbuckle JH, Wang C-R, Kristie TM, Rehermann B, Trinchieri G, Brenchley JM, O'Shea JJ, Belkaid Y. Non-classical Immunity Controls Microbiota Impact on Skin Immunity and Tissue Repair. Cell [Internet]. [cited 2018 Jan 25]; Available from: https://www.sciencedirect.com/science/article/ pii/S0092867417315131

11. DESMAN: a new tool for de novo extraction of strains from metagenomes | Genome Biology | Full Text [Internet]. [cited 2020 Mar 23]. Available from: https://doi.org/10.1186/s13059-017-1309-9

12. Truong DT, Tett A, Pasolli E, Huttenhower C, Segata N. Microbial strain-level population structure and genetic diversity from metagenomes. Genome Res. 2017;gr.216242.116. PMID: 28167665

13. Luo C, Knight R, Siljander H, Knip M, Xavier RJ, Gevers D. ConStrains identifies microbial strains in metagenomic datasets. Nat Biotechnol. 2015; 33(10):1045-52. https://doi.org/10.1038/nbt.3319.

14. Structure, Function and Diversity of the Healthy Human Microbiome. Nature. 2012;486(7402):207-214. PMCID: PMC3564958.

15. Oh J, Byrd AL, Park M, Kong HH, Segre JA. Temporal stability of the human skin microbiome. Cell. 2016;165(4):854-66. https://doi.org/10.1016/j.cell.2016. 04.008 .

16. Lloyd-Price J, Mahurkar A, Rahnavard G, Crabtree J, Orvis J, Hall AB, Brady A, Creasy HH, McCracken C, Giglio MG, McDonald D, Franzosa EA, Knight R, White $\mathrm{O}$, Huttenhower C. Strains, functions and dynamics in the expanded Human Microbiome Project. Nature [Internet]. 201720 [cited 2017 Sep 20]; advance online publication. Available from: http://www.nature.com/nature/ journal/vaop/ncurrent/full/nature23889.html?foxtrotcallback=true

17. Donaldson GP, Lee SM, Mazmanian SK. Gut biogeography of the bacterial microbiota. Nat Rev Microbiol. 2016;14(1):20-32. PMCID: PMC4837114.

18. Deo P, Deshmukh R. Oral microbiome: unveiling the fundamentals. J Oral Maxillofac Pathol. 2019;23(1):122-8. https://doi.org/10.4103/jomfp.JOMFP_304_18.

19. Costalonga M, Herzberg MC. The oral microbiome and the immunobiology of periodontal disease and caries. Immunol Lett. 2014;162(2 Pt A):22-38. PMCID: PMC4346134.

20. Oh J, Byrd AL, Deming C, Conlan S, NISC Comparative Sequencing Program, Kong $\mathrm{HH}$, Segre JA. Biogeography and individuality shape function in the human skin metagenome. Nature. 2014;514(7520):59-64. PMCID: PMC4185404.

21. Lagier J-C, Khelaifia S, Alou MT, Ndongo S, Dione N, Hugon P, et al. Culture of previously uncultured members of the human gut microbiota by culturomics. Nat Microbiol. 2016;1(12):1-8. 
22. Lagier J-C, Hugon P, Khelaifia S, Fournier P-E, Scola BL, Raoult D. The rebirth of culture in microbiology through the example of Culturomics to study human gut microbiota. Clin Microbiol Rev 2015;28(1):237-264. PMID: 25567229, DOl: https://doi.org/10.1128/CMR.00014-14

23. Lagier J-C, Armougom F, Million M, Hugon P, Pagnier I, Robert C, Bittar F, Fournous G, Gimenez G, Maraninchi M, Trape J-F, Koonin EV, La Scola B, Raoult D. Microbial culturomics: paradigm shift in the human gut microbiome study. Clin Microbiol Infect Off Publ Eur Soc Clin Microbiol Infect Dis 2012;18(12):1185-1193. PMID: 23033984, DOI: https://doi.org/1 $0.1111 / 1469-0691.12023$

24. Berdy B, Spoering AL, Ling LL, Epstein SS. In situ cultivation of previously uncultivable microorganisms using the ichip. Nat Protoc. 2017;12(10):223242. https://doi.org/10.1038/nprot.2017.074

25. Cross KL, Campbell JH, Balachandran M, Campbell AG, Cooper SJ, Griffen A, et al. Targeted isolation and cultivation of uncultivated bacteria by reverse genomics. Nat Biotechnol. 2019;37(11):1314-21. https://doi.org/10.1038/s41 587-019-0260-6.

26. Forster SC, Kumar N, Anonye BO, Almeida A, Viciani E, Stares MD, et al. A human gut bacterial genome and culture collection for improved metagenomic analyses. Nat Biotechnol. 2019;37(2):186-92. https://doi.org/1 0.1038/s41587-018-0009-7.

27. Browne HP, Forster SC, Anonye BO, Kumar N, Neville BA, Stares MD, et al. Culturing of 'unculturable' human microbiota reveals novel taxa and extensive sporulation. Nature. 2016;533(7604):543-6. https://doi.org/10.1038/ nature17645.

28. 1,520 reference genomes from cultivated human gut bacteria enable functional microbiome analyses | Nature Biotechnology [Internet]. [cited 2019 Feb 4]. Available from: https://www.nature.com/articles/s41587-0180008-8\#MOESM3

29. Evans CA, Smith WM, Johnston EA, Giblett ER. Bacterial Flora of the Normal human skin*. J Invest Dermatol. 1950;15(4):305-24. https://doi.org/10.1038/ jid.1950.105.

30. Bossard DA, Ledergerber B, Zingg PO, Gerber C, Zinkernagel AS, Zbinden R, Achermann Y. Optimal length of cultivation time for isolation of Propionibacterium acnes in suspected bone and joint infections is more than 7 days. Patel R, editor. J Clin Microbiol 2016;54(12):3043-3049, DOI: https://doi.org/10.1128/JCM.01435-16.

31. Myles IA, Reckhow JD, Williams KW, Sastalla I, Frank KM, Datta SK. A method for culturing gram-negative skin microbiota. BMC Microbiol. 2016;16(1):60. https://doi.org/10.1186/s12866-016-0684-9.

32. Burton JP, Wescombe PA, Cadieux PA, Tagg JR. Beneficial microbes for the oral cavity: time to harness the oral streptococci? Benef Microbes. 2011;2(2): 93-101. PMID: 21840808. https://doi.org/10.3920/BM2011.0002.

33. Bacic MK, Smith CJ. Laboratory maintenance and cultivation of bacteroides species. Curr Protoc Microbiol. 2008; Chapter 13:Unit 13C.1. PMCID: PMC3836205

34. Baron EJ. Approaches to identification of anaerobic Bacteria. Man Clin Microbiol Elev Ed. 2015;1:905-8. https://doi.org/10.1128/9781555817381. ch50.

35. Marsh PD, Lewis MAO, Rogers H, PhD DDW BSC (Hons), Wilson M. Marsh and Martin's Oral Microbiology - E-Book. Elsevier Health Sciences; 2016.

36. Jousimies-Somer H, Sutter VL. Wadsworth-KTL anaerobic bacteriology manual. Belmont: Star Pub; 2002.

37. Balows A, Trüper HG, Dworkin M, Harder W, Schleifer K-H, editors. The Prokaryotes: A Handbook on the Biology of Bacteria: Ecophysiology, Isolation, Identification, Applications [Internet]. 2nd ed. New York: SpringerVerlag; 1992 [cited 2021 Mar 5]. Available from: https://www.springer.com/ gp/book/9781475721911

38. Zhou X, Li Y. Atlas of Oral Microbiology: From Healthy Microflora to Disease. Academic Press; 2015.

39. Nolte WA. Oral microbiology: with basic microbiology and immunology. 4th ed. St. Louis: Mosby; 1982.

40. Seymour GJ, Cullinan MP, Heng NCK, editors. Oral Biology: Molecular Techniques and Applications [Internet]. Humana Press; 2010 [cited $2021 \mathrm{Mar}$ 5]. Available from: https://www.springer.com/gp/book/9781607618195

41. Kong HH, Andersson B, Clavel T, Common JE, Jackson SA, Olson ND, et al. Performing skin microbiome research: a method to the madness. J Invest Dermatol. 2017 Mar;137(3):561-8. https://doi.org/10.1016/j.jid.2016.10.033.

42. Hirvonen JJ, Kerttula A-M, Kaukoranta S-S. Performance of SaSelect, a Chromogenic Medium for Detection of Staphylococci in Clinical Specimens. J Clin Microbiol. 2014;52(4):1041-1044. PMCID: PMC3993506.
43. Bastos M Do C De F, Coutinho BG, Coelho MLV. Lysostaphin: A Staphylococcal Bacteriolysin with Potential Clinical Applications. Pharmaceuticals. 2010;3(4):1139-1161. PMCID: PMC4034026.

44. Wang Y, Kao M-S, Yu J, Huang S, Marito S, Gallo RL, et al. A precision microbiome approach using sucrose for selective augmentation of Staphylococcus epidermidis fermentation against Propionibacterium acnes. Int J Mol Sci. 2016;17(11):1870. https://doi.org/10.3390/ijms1 7111870 .

45. Adams E. The antibacterial action of crystal violet*. J Pharm Pharmacol. 1967;19(12):821-6. https://doi.org/10.1111/j.2042-7158.1967.tb09550.x.

46. Riegel $P$, Heller R, Prevost $G$, Jehl F, Monteil $H$. Corynebacterium durum sp. nov., from Human Clinical Specimens. Int J Syst Evol Microbiol. 1997;47(4): 1107-11.

47. Li Y, Shan M, Zhu Z, Mao X, Yan M, Chen Y, et al. Application of MALDI-TOF MS to rapid identification of anaerobic bacteria. BMC Infect Dis. 2019 Nov 7; 19(1):941. https://doi.org/10.1186/s12879-019-4584-0.

48. Veen SQ van, Claas ECJ, Kuijper EJ. High-Throughput Identification of Bacteria and Yeast by Matrix-Assisted Laser Desorption Ionization-Time of Flight Mass Spectrometry in Conventional Medical Microbiology Laboratories. J Clin Microbiol. American Society for Microbiology Journals; 2010;48(3):900-907. PMID: 20053859

49. Truong DT, Franzosa EA, Tickle TL, Scholz M, Weingart G, Pasolli E, et al. MetaPhIAn2 for enhanced metagenomic taxonomic profiling. Nat Methods. 2015 Oct;12(10):902-3. https://doi.org/10.1038/nmeth.3589.

50. Maiden MCJ, Jansen van Rensburg MJ, Bray JE, Earle SG, Ford SA, Jolley KA, McCarthy ND. MLST revisited: the gene-by-gene approach to bacterial genomics. Nat Rev Microbiol. 2013;11(10):728-736. PMCID: PMC3980634.

51. Microbial DNA Typing by Automated Repetitive-Sequence-Based PCR [Internet]. Journal of Clinical Microbiology. [cited 2021 Jun 3]. Available from: https://doi.org/10.1128/JCM.43.1.199-207.2005

52. Versalovic J, de Bruijn FJ, Lupski JR. Repetitive Sequence-based PCR (rep$P(R)$ DNA fingerprinting of bacterial genomes. In: de Bruijn FJ, Lupski JR, Weinstock GM, editors. Bact genomes Phys Struct anal [internet]. Boston, MA: springer US; 1998 [cited 2021 Jun 3]. p. 437-454. Available from: https://doi.org/10.1007/978-1-4615-6369-3_34, 1998.

53. Novais Â, Freitas AR, Rodrigues C, Peixe L. Fourier transform infrared spectroscopy: unlocking fundamentals and prospects for bacterial strain typing. Eur J Clin Microbiol Infect Dis Off Publ Eur Soc Clin Microbiol 2019; 38(3):427-448. PMID: 30483997, DOl: https://doi.org/10.1007/s10096-01 8-3431-3

54. Zhao S, Lieberman TD, Poyet M, Kauffman KM, Gibbons SM, Groussin M, Xavier RJ, Alm EJ. Adaptive Evolution within Gut Microbiomes of Healthy People. Cell Host Microbe. 2019; PMID: 31028005

55. Tomida S, Nguyen L, Chiu B-H, Liu J, Sodergren E, Weinstock GM, Li H. PanGenome and Comparative Genome Analyses of Propionibacterium acnes Reveal Its Genomic Diversity in the Healthy and Diseased Human Skin Microbiome. mBio [Internet]. 2013 Apr 30 [cited 2015 Sep 18];4(3). Available from: http://www.ncbi.nlm.nih.gov/pmc/articles/PMC3663185/ PMCID: PMC3663185.

56. Buffalo V. A 3'-end adapter contaminant trimmer [Internet]. 2021 [cited 2021 Jun 3]. Available from: https://github.com/vsbuffalo/scythe

57. Josh N, Fass J. Sickle: A sliding-window, adaptive, quality-based trimming tool for FastQ files (Version 1.33) [Software]. Available Httpsgithubcomnajoshisickle. 2011;

58. Langmead B, Salzberg SL. Fast gapped-read alignment with bowtie 2. Nat Methods. 2012 Apr;9(4):357-9. https://doi.org/10.1038/nmeth.1923.

59. Li H, Handsaker B, Wysoker A, Fennell T, Ruan J, Homer N, Marth G, Abecasis G, Durbin R, 1000 Genome Project Data Processing Subgroup. The Sequence Alignment/Map format and SAMtools. Bioinforma Oxf Engl. 2009; 25(16):2078-2079. PMCID: PMC2723002.

60. Altschul SF, Gish W, Miller W, Myers EW, Lipman DJ. Basic local alignment search tool. J Mol Biol. 1990;215(3):403-10. https://doi.org/10.1016/S0022-2 836(05)80360-2.

61. Bankevich A, Nurk S, Antipov D, Gurevich AA, Dvorkin M, Kulikov AS, Lesin VM, Nikolenko SI, Pham S, Prjibelski AD, Pyshkin AV, Sirotkin AV, Vyahhi N, Tesler G, Alekseyev MA, Pevzner PA. SPAdes: A New Genome Assembly Algorithm and Its Applications to Single-Cell Sequencing. J Comput Biol. 2012;19(5):455-477. PMCID: PMC3342519.

62. Chaumeil P-A, Mussig AJ, Hugenholtz P, Parks DH. GTDB-Tk: a toolkit to classify genomes with the Genome Taxonomy Database. Bioinforma Oxf Engl. 2019; PMCID: PMC7703759. 
63. Price MN, Dehal PS, Arkin AP. FastTree 2 - Approximately MaximumLikelihood Trees for Large Alignments. PLoS ONE [Internet]. 2010 Mar 10 [cited 2021 Feb 24];5(3). Available from: https:/www.ncbi.nlm.nih.gov/ pmc/articles/PMC2835736/ PMCID: PMC2835736.

64. Molecular Evolution, Phylogenetics and Epidemiology [Internet]. [cited 2021 Feb 24]. Available from: http://tree.bio.ed.ac.uk/

\section{Publisher's Note}

Springer Nature remains neutral with regard to jurisdictional claims in published maps and institutional affiliations.

Ready to submit your research? Choose BMC and benefit from:

- fast, convenient online submission

- thorough peer review by experienced researchers in your field

- rapid publication on acceptance

- support for research data, including large and complex data types

- gold Open Access which fosters wider collaboration and increased citations

- maximum visibility for your research: over $100 \mathrm{M}$ website views per year

At $\mathrm{BMC}$, research is always in progress.

Learn more biomedcentral.com/submissions 